\title{
Um olhar sobre a educação inclusiva no Brasil por meio do BDTD
}

\author{
A look at inclusive education in Brazil through the BDTD \\ Una mirada a la educación inclusiva em Brasil a través del BDTD
}

Recebido: 01/08/2021 | Revisado: 04/08/2021 | Aceito: 05/08/2021 | Publicado: 10/08/2021

\author{
Débora Lisboa Correa Costa \\ ORCID: https://orcid.org/0000-0002-9541-5465 \\ Universidade Federal do Estado do Pará, Brasil \\ E-mail: debylisboa2007@yahoo.com.br \\ Andreia Cristina da Silva Costa \\ ORCID: https://orcid.org/0000-0002-1720-4945 \\ Universidade do Estado do Pará, Brasil \\ E-mail: crisandreia2010@gmail.com \\ Anderson da Silva Costa \\ ORCID: https://orcid.org/0000-0002-3955-1817 \\ Biodiversidade e Biotecnologia da Amazônia Legal-BIONORTE, Brasil \\ E-mail: anderson.costa@embrapa.br
}

\begin{abstract}
Resumo
O presente estudo tem como objetivo quantificar as produções acadêmicas stricto sensu que constam na Biblioteca Digital Brasileira de Teses e Dissertações (BDTD) que abordam o tema Educação Inclusiva. O propósito do levantamento foi elaborar um panorama do número de produções científicas sobre a temática nos últimos vinte anos. Para tal, este estudo utilizou a revisão bibliográfica, onde foi realizado o levantamento de produções strito sensu no site do BDTD, utilizando-se o descritor Educação Inclusiva e Crianças, entre aspas, com os filtros dissertações e teses no período de 2000 a 2020. O estudo registrou um total de trezentos e quatorze produções científicas com essa temática, sendo 235pesquisas classificadas na modalidade de Mestrado acadêmico e 79 teses de Doutorado. Os dados revelaram que o ano de 2018 foi período que registrou maior concentração dessa temática com 36 inserções de pesquisas no ramo, seguido do ano de 2017, que apontou 31 ocorrências. Em média essa temática registrou 15 publicações por ano de ocorrência ao longo da série temporal, demonstrando um grande volume de produção Strito Sensu sobre o tema no Brasil. As Universidades que mais se destacaram nas produções dessa temática foram UNESP com 36 ocorrências, seguida da UFSCAR com 32 produções e a PUC com 27 ocorrências. A análise apontou que as regiões que mais produziram sobre o assunto foi a região sudeste do país e de menor índice foi a região nordeste.
\end{abstract}

Palavras-chave: Educação inclusiva; Crianças; BDTD; Produção acadêmica.

\begin{abstract}
This study aims to quantify the stricto sensu academic productions contained in the Brazilian Digital Library of Theses and Dissertations (BDTD) that address the theme Inclusive Education. The purpose of the survey was to elaborate an overview of the number of scientific productions on the subject in the last twenty years. To this end, this study used a bibliographic review, where a survey of stricto sensu productions was carried out on the BDTD website, using the descriptor Inclusive Education and Children, in quotation marks, with filters dissertations and theses in the period 2000 to 2020. The study registered a total of three hundred and fourteen scientific productions with this theme, with 235 researches classified in the academic Masters modality and 79 Doctoral theses. The data revealed that the year 2018 was the period that recorded the highest concentration of this theme with 36 insertions of research in the field, followed by the year 2017, which showed 31 occurrences. On average, this theme registered 15 publications per year of occurrence throughout the time series, demonstrating a large volume of Srito Sensu production on the subject in Brazil. The Universities that stood out the most in the productions on this theme were UNESP with 36 occurrences, followed by UFSCAR with 32 productions and PUC with 27 occurrences. The analysis pointed out that the regions that produced the most on the subject were the southeast region of the country and with the lowest index was the northeast region.
\end{abstract}

Keywords: Inclusive education; Kids; BDTD; Academic production.

\section{Resumen}

Este estudio tiene como objetivo cuantificar las producciones académicas stricto sensu contenidas en la Biblioteca Digital Brasileña de Tesis y Disertaciones (BDTD) que abordan el tema de la Educación Integrada. El propósito de la encuesta fue elaborar un panorama del número de producciones científicas sobre el tema en los últimos veinte años. Para ello, este estudio utilizó una revisión de literatura, donde se realizó una encuesta de producciones stricto sensu en 
el sitio web del BDTD, utilizando el descriptor Educación Inclusiva e Infancia, entre comillas, con filtros disertaciones y tesis en el período 2000 a 2020. El estudio registró un total de trescientas catorce producciones científicas con esta temática, con 235 investigaciones clasificadas en la modalidad de Maestría académica y 79 tesis doctorales. Los datos revelaron que el año 2018 fue el período que registró la mayor concentración de este tema con 36 inserciones de investigación en el campo, seguido por el año 2017, que mostró 31 ocurrencias. En promedio, este tema registró 15 publicaciones por año de ocurrencia a lo largo de la serie temporal, lo que demuestra un gran volumen de producción de Srito Sensu sobre el tema en Brasil. Las Universidades que más se destacaron en las producciones sobre este tema fueron UNESP con 36 ocurrencias, seguida de UFSCAR con 32 producciones y PUC con 27 ocurrencias. El análisis señaló que las regiones que más produjeron en el tema fueron la región sureste del país y con el índice más bajo fue la región noreste.

Palabras clave: Educación inclusiva; Niños; BDTD; Producción académica.

\section{Introdução}

No Brasil, a educação inclusiva manifestou suas primeiras ações a partir dos anos 1980, com a divulgação de dados preocupantes sobre o fracasso, a evasão, a repetência escolar, e o aumento da procura pela criação de escolas especiais. Lourenço (2010) resgata que os movimentos sociais colaboraram grandemente para a ampliação das práticas inclusivas ao exigir que as instituições sociais extinguissem as barreiras que mantinham as pessoas com necessidades especiais afastadas das atividades escolares e que a partir deste momento as instituições educacionais deveriam proporcionar a aprendizagem deste público.

Barbosa et al., (2018) complementa que a partir destes movimentos liderados por organismos internacionais como a Organização das Nações Unidas (ONU), Organização das Nações Unidas para a Educação, a Ciência e a Cultura (Unesco), Organização dos Estados Americanos (OEA), Fundo das Nações Unidas para a Infância (Unicef) e Banco Mundial (BM), o Brasil foi impulsionado a rever suas políticas públicas educacionais, para que houvesse o envolvimento de pessoa com deficiência fossem atendidos bem como que o tema fosse tratado pelas Universidades, especificamente no curso de Pedagogia.

A preocupação com a inclusão de crianças especiais no cenário educacional brasileiro teve continuidade com o surgimento do Estatuto da Criança e Adolescente (ECA), criado pela Lei 8.069/1990 ao assegurar a todas as crianças “a igualdade de condições para o acesso e permanência na escola, o direito de ser respeitado por seus professores e o acesso à escola pública e gratuita próxima de sua residência" (Art. 35). Diante do que estabelece o ECA, existe o desafio da realidade enfrentada pelas escolas públicas brasileiras de cumprirem a oferta de escolarização a todos os seus alunos, tendo em vista que em sua maioria, não existe estrutura física adequada e uma quantidade satisfatória de corpo técnico especializado para atendê-los nos ambientes escolares.

A Educação Especial que por muito tempo configurou-se como um sistema paralelo de ensino, vem redimensionando o seu papel, antes restrito ao atendimento direto dos educandos especiais, para atuar, prioritariamente como suporte à escola regular no recebimento deste alunado (Sassaki, 1997). Sobre o assunto, Neto et al., (2018) realizou um resgate histórico do movimento da educação especial até a implementação da educação inclusiva no ensino regular e constatou que embora existam avanços políticos, na prática ainda há a necessidade de disponibilizar um ambiente apropriado para o tipo e o nível de deficiência de seus alunos.

Para Laplane (2006) aponta a dificuldade de implementar políticas de educação inclusiva ao afirmar que ainda permanece uma larga brecha entre tal política e a prática nas escolas brasileiras. Sobre o assunto, o autor acrescenta que existe uma contradição no âmbito educacional diante do que prevê a legislação da Educação Inclusiva e à tendência excludente vivenciada pelos discentes com necessidades especiais.

Atualmente, a Educação Inclusiva é norteada pela Política Nacional de Educação Especial na Perspectiva da Educação Inclusiva (PNEEPEI 2008) que determina que os alunos especiais sejam incluídos em escolas regulares para assistirem aula junto aos demais discentes: 
[...] os sistemas de ensino devem assegurar aos alunos currículo, métodos, recursos e organização específicos para atender às suas necessidades; assegura a terminalidade específica àqueles que não atingiram o nível exigido para a conclusão do ensino fundamental, em virtude das suas deficiências (Brasil, 2008, p. 2).

Considerando PNEEPEI (2008), compreende-se que a inclusão é um processo que exige adaptação da escola e a conscientização de todo o corpo escolar para que os alunos especiais não permaneçam somente fisicamente neste espaço, porém, tenham condições de desenvolver plenamente sua aprendizagem. Mantoan (2006) afirma que a inclusão engloba modernização e reestruturação das condições da maioria de nossas escolas, uma vez que as dificuldades desses alunos não são apenas deles, mas procedem do modo como o ensino é ministrado e de como ocorre a aprendizagem.

Ferreira (2006) explica que a inclusão aponta à necessidade de reconfigurar as possibilidades de interação das pessoas com deficiências, atendendo as exigências especificas que se apresentam para concretização da aprendizagem, utilizados métodos para que essa necessidade seja amenizada. Assim, é importante notar que a proposta de inclusão leva à transformação de toda a estrutura educacional, no intuito de valorizar as diferenças de todos os sujeitos que vivenciam o meio escolar e como ponto primordial para a construção de uma consciência social mais sensível e mais humana (Albuquerque, 2005). Além destes aspectos, Silva et al., (2020) destaca a necessidade de capacitar professores para atuarem em seus campos tão específicos:

[...] repensar as questões de inclusão em nossa sociedade é fundamental e, sobretudo, essa discussão precisa estar presente nos processos formativos de docentes, possibilitando ampliar a noção de inclusão para os desdobramentos das políticas vigentes nos contextos educacionais, nos quais os professores são os principais articuladores de estratégias para atender à demanda (Silva et al., 2020, p. 13).

Apesar dos avanços da Educação Inclusiva nas Políticas Educacionais Brasileiras Laplane (2006) relembra que ainda existem lacunas que precisam ser preenchidas para alcançarmos uma coerência entre a teoria e a prática dentro do assunto, tal necessidade pode ser ajustada ao conhecermos o atual cenário das produções cientificas sobre a temática. Nesse sentido autores alertam que o tema requer:

[...] uma investigação do seu percurso ao longo dos anos, pois as concepções que se tem sobre a deficiência vêm sendo construídas e transformadas ao longo da história" (Miranda, 2019, p. 12).

Estudos mostram que a implementação da educação inclusiva precisa ser ajustada para atender a sua proposta nos diversos níveis de ensino, perpassando pela escola básica até o ensino superior. Sobre este último nível, temos a pesquisa de Gomes et al., (2021) no qual realizaram uma revisão sistemática sobre o tema, apontam em seus resultados que é necessário a adequação das salas de aula e disponibilização de material didático necessário.

Neste sentido Dupin e Silva (2020), complementam sobre a temática, apontando nos resultados de sua pesquisa que embora a educação inclusiva possui leis, decretos e políticas que se complementam porém ainda encontram-se em evolução através das atuais conquistas das pessoas com deficiência na sociedade bem como na conquista por seus direitos.

Segundo Almeida et al., (2020) ao investigar o perfil docente e o modo como professores atuantes na área de Ciências do Ensino Regular, dos níveis Fundamental e Médio, de escolas públicas da rede municipal e estadual, no interior do Amazonas, apontou através dos resultados obtidos que ainda existe uma necessidade de refletir e de agir sobre educação inclusiva, tanto no aspecto de acesso físico e educacional de alunos, quanto no relacionado à formação dos professores.

Durante as aulas presenciais os alunos especiais apresentavam dificuldades para acompanhar o ensino que estava sendo ministrado e sua aprendizagem recebe um desafio ainda maior ao consideramos o surgimento da pandemia do COVID-19, onde o aluno não contara com a presença do professor em sala. O recente estudo de Borges, Bandeira e Corrêa (2020) alerta que ao analisarem o desempenho de alunos especiais em plataformas digitais constataram que eles não apresentaram domínio dos 
programas digitais e, portanto, concluem que nessa fase pandêmica esses alunos continuam sem condições apropriadas para o desenvolvimento de sua aprendizagem.

Nesse sentido, este estudo pretende apresentar um panorama das pesquisas atuais sobre a Educação Inclusiva no Brasil por meio de levantamento bibliográfico.

\section{Metodologia}

Destaca-se que é de interesse deste estudo analisar as dissertações de mestrado e as teses de doutorado produzidas nos programas de pós-graduação stricto sensu das universidades brasileiras por permitirem a caracterização e a produção científica da Educação Inclusiva.

Esta pesquisa bibliográfica utiliza a abordagem integrada, definida por Severino (2017), como estudos que envolvem a análise de dados quantitativos e qualitativos simultaneamente. Nesse sentido, ressalta-se que inicialmente durante o mês de junho de 2021, foi realizado o mapeamento do número de produções cientificas (strito sensu), provenientes de mestrados acadêmicos e doutorados inseridos na plataforma da Biblioteca Brasileira de Teses e Dissertações (BDTD). Após esse levantamento, foi delimitado a base temporal do acervo, onde o material selecionado foi restringido a trabalhos publicados nos últimos 20 anos no Brasil. Para a seleção das produções strito sensu, foi utilizado os descritores "Educação Inclusiva" e "crianças" na base de busca da plataforma da BDTD. Em seguida, foi aplicado o filtro temporal de todas as produções publicadas, no período de 2000 a 2020. Para a análise do conteúdo das produções selecionadas adotamos Bardin (2016).

\section{Resultados e Discussões}

Os resultados deste levantamento apontaram que a maior produção Stricto Sensu sobre Educação Inclusiva obtida por meio da Base de dados BDTD, foi em nível de Mestrado Acadêmico, totalizando 235 publicações. Este percentual representa aproximadamente $75 \%$ do valor total das 314 publicações. No que diz respeito aos números de ocorrências dessa temática em nível de Doutorado, constatou-se que existem apenas 79 teses que abordam a Educação Inclusiva (Figura 1).

Figura 1. Distribuição das publicações Stricto Sensu a partir dos dados BDTD.

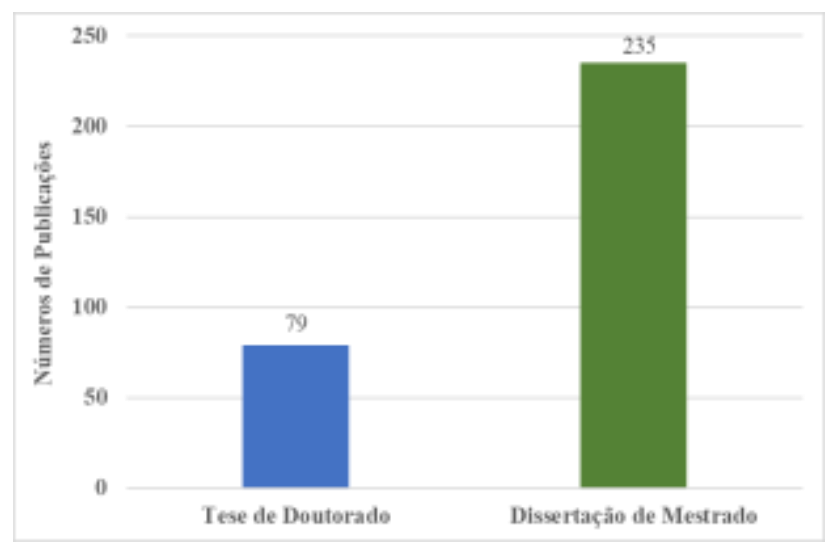

Fonte: Base das publicações BDTD.

Os dados demonstram que o país dispõe de profissionais qualificados com essa temática, o que possibilita aos alunos com deficiência ou outra especificidade, partilhar do mesmo espaço social educacional que os demais com acompanhamento especializado. Para Cavalleiro (2006, p. 21): "Silenciar-se diante do problema não apaga magicamente as diferenças, e ao contrário, permite que cada um construa, a seu modo, um entendimento muitas vezes estereotipado do outro que lhe é diferente". É imprescindível, portanto, reconhecer esse problema e combatê-lo no espaço escolar. 
Para que isso aconteça os docentes precisam urgentemente ter qualificações acadêmicas em níveis de Mestrado e Doutorado como pode - se afirmar, em termos amplos o estudo de Gonzalez (2002), que aponta que todo discente é aluno com necessidades educativas especiais, já que possui características individuais que o diferenciam dos demais e necessita de uma educação condizente com as mesmas. Neste sentido, ter uma educação harmônica é dispor de um profissional capacitado nos três níveis acadêmicos (graduação, mestrado e doutorado).

O total de 314 publicações científica (teses e dissertações), corrobora com a literatura especializada ao demonstrar que a discussão sobre essa temática é relativamente nova e precisa ser mais explorada nas escolas e nas universidades brasileiras, principalmente em nível de doutorado, pois a tese é considerada o tipo mais representativo do trabalho científico (Severino, 2017).

Analisando a janela temporal de 20 anos (2000 a 2020), percebe-se um crescimento dessa temática a partir do ano de 2006, sendo representado por 12 produções. Entretanto, com o passar do tempo, os dados revelam que houve uma queda acentuada em 2011, resumido a uma pequena amostra de 8 produções. Em seguida, a figura 2 mostra que o tema volta a ser discutido com maior frequência nos programas de pós-graduação strito sensu, tendo um novo crescimento no ano de 2012, representado por 25 ocorrências. Por fim, ao analisar as inserções referentes ao ano de 2018, nota-se um crescimento considerável do número de publicações, com 36 produções acadêmicas (Figura 2).

Figura 2. Distribuição das publicações Stricto Sensu a partir dos dados BDTD ao longo da série temporal.

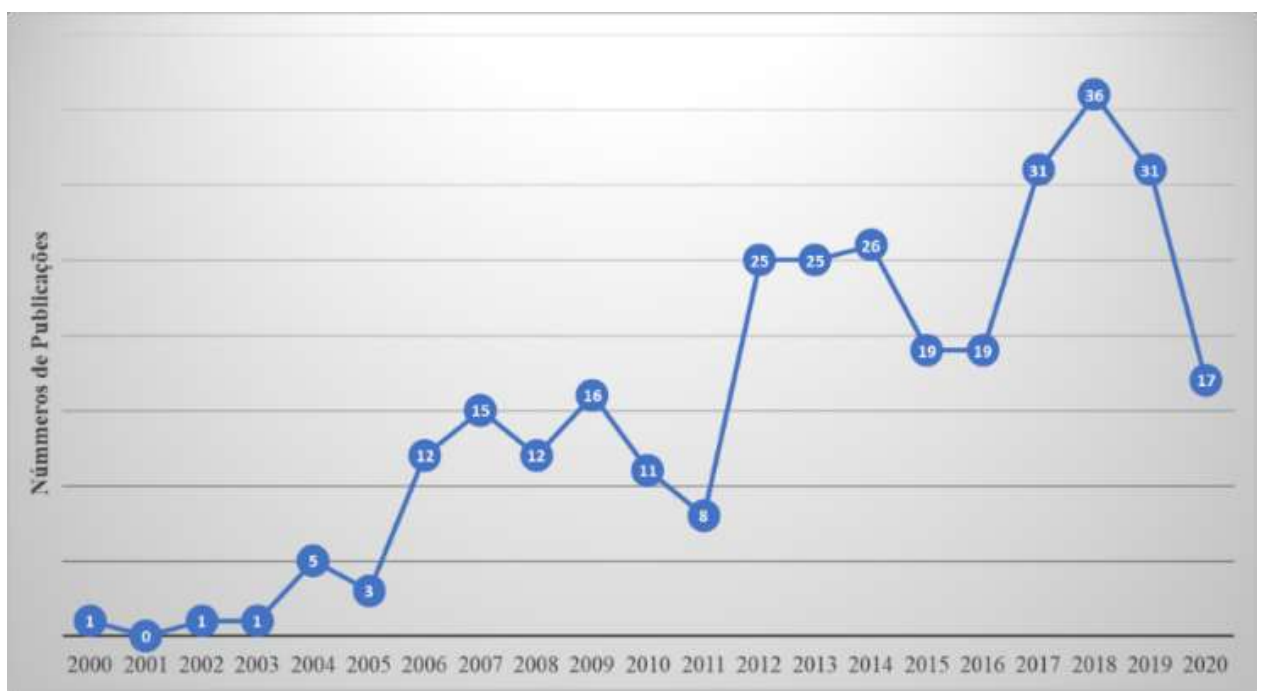

Fonte: Base das publicações BDTD.

Ao analisar a ranking das universidades que mais concentraram publicações com essa temática, $50 \%$ dessa temática se concentrou-se em 7 Universidades, destaca-se a Universidade Estadual Paulista- UNESP, com 36 publicações. Em segundo lugar, aparece a Universidade Federal de São Carlos- UFSCAR, com 32 ocorrências; a Pontifícia Universidade Católica de São Paulo- PUC-SP, com 27 publicações; seguida pela Universidade de São Paulo - USP, representada por 22 produções; a Universidade de Brasília - UNB e por fim, o menor índice, com a Universidade Federal do Amazonas- UFAM, apresentando 10 publicações sobre o tema, conforme é possível acompanhar na Figura 3.

Destaca-se que conforme os dados acima, é possível perceber que as regiões que mais produziram sobre o assunto foi a região sudeste do país e de menor índice foi a região nordeste. 
Figura 3. Distribuição das publicações Stricto Sensu a partir dos dados BDTD ao longo das Universidades Brasileiras.

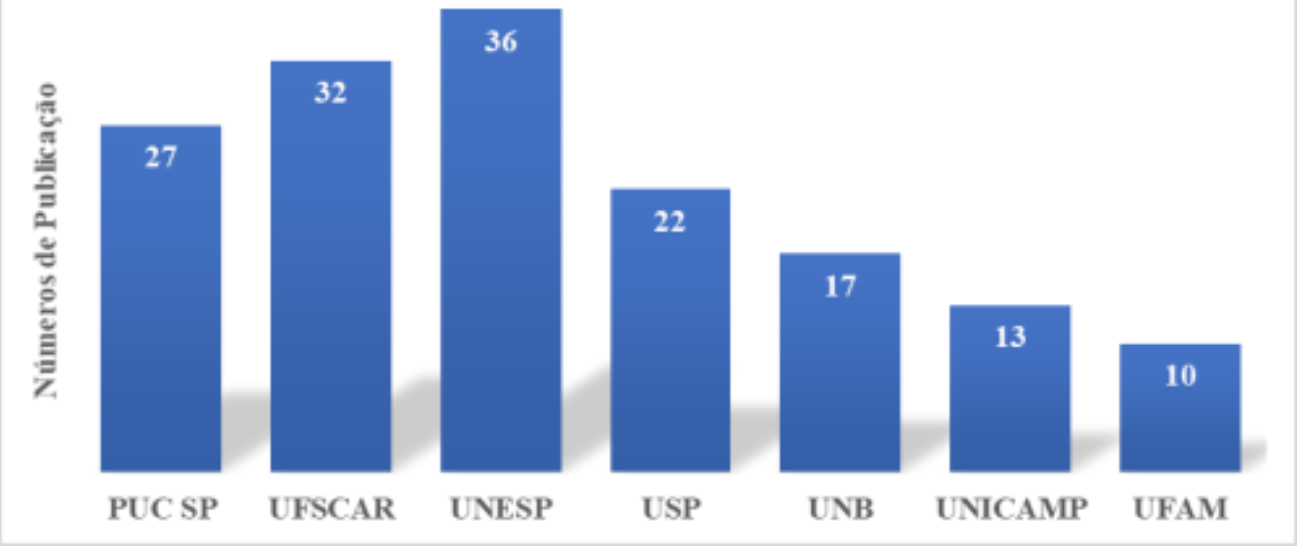

Fonte: Base das publicações BDTD.

Educação inclusiva é o processo que ocorre em escolas de qualquer nível preparadas para propiciar um ensino de qualidade a todos os alunos independentemente de seus atributos pessoais, inteligências, estilos de aprendizagem e necessidades comuns ou especiais. A inclusão escolar é uma forma de inserção em que a escola comum tradicional é modificada para ser capaz de acolher qualquer aluno incondicionalmente e de propiciar-lhe uma educação de qualidade.

Neste sentido, podemos destacar também que a Universidade Federal de São Carlos- UFSCAR, que aparece em segundo lugar com 32 produções acadêmicas, foi a Universidade que mais produziu teses de doutorado com essa temática seguida da USP com 10 teses (Tabela 1).

Vale destacar a diferença fundamental em relação à tese de doutorado está no caráter de originalidade do trabalho. Segundo Matczak (1971), trata-se de uma pesquisa ainda vinculada a uma fase de iniciação à ciência, de um exercício diretamente orientado, primeira manifestação de um trabalho pessoal de pesquisa, não se pode exigir da dissertação de mestrado o mesmo nível de originalidade e o mesmo alcance de contribuição ao progresso e desenvolvimento da ciência em questão.

Tabela 1. Distribuição da Produções Cientificas sobre a Temática nas Universidades.

\begin{tabular}{l|c|c|c}
\hline Universidades & Teses de Doutorado & $\begin{array}{c}\text { Dissertação de } \\
\text { Mestrado }\end{array}$ & Total Geral \\
\hline FDV & & 1 & 1 \\
\hline MACKENZIE & 2 & 2 & 4 \\
\hline METODISTA & & 4 & 1 \\
\hline PUC/GO & 7 & 20 & 27 \\
\hline PUC/SP & & 2 & 2 \\
\hline UCPEL & & 3 & 3 \\
\hline UCS & 1 & & 1 \\
\hline UCSAL & & 1 & 2 \\
\hline UEL & 1 & 1 & 3 \\
\hline UEM & 1 & 2 & 2 \\
\hline UEPB & & 2 & 1 \\
\hline UEPG & & 1 & 2 \\
\hline UERJ & & 2 & 2 \\
\hline UFAL & & & 2 \\
\hline
\end{tabular}


Research, Society and Development, v. 10, n. 10, e282101018961, 2021

(CC BY 4.0) | ISSN 2525-3409 | DOI: http://dx.doi.org/10.33448/rsd-v10i10.18961

\begin{tabular}{|c|c|c|c|}
\hline UFAM & 1 & 9 & 10 \\
\hline UFC & 3 & 7 & 10 \\
\hline UFES & 1 & 6 & 7 \\
\hline UFG & 1 & 7 & 8 \\
\hline UFGD & 1 & 7 & 8 \\
\hline UFJF & & 8 & 8 \\
\hline UFMA & & 2 & 2 \\
\hline UFMG & 2 & 7 & 9 \\
\hline UFMS & & 1 & 1 \\
\hline UFMT & & 3 & 3 \\
\hline UFPA & 1 & 8 & 9 \\
\hline UFPB & & 5 & 5 \\
\hline UFPE & 1 & 4 & 5 \\
\hline UFPEL & & 1 & 1 \\
\hline UFPR & 2 & 7 & 9 \\
\hline UFRGS & 3 & 4 & 7 \\
\hline UFRN & 1 & 2 & 3 \\
\hline UFRPE & & 1 & 1 \\
\hline UFS & 1 & 2 & 3 \\
\hline UFSC & & 7 & 7 \\
\hline UFSCAR & 22 & 10 & 32 \\
\hline UFSM & & 2 & 2 \\
\hline UFTM & 1 & 1 & 2 \\
\hline UFV & & 1 & 1 \\
\hline UNB & 4 & 13 & 17 \\
\hline UNESC & & 1 & 1 \\
\hline UNESP & 8 & 28 & 36 \\
\hline UNICAMP & 2 & 11 & 13 \\
\hline UNICAP & & 1 & 1 \\
\hline UNIFAL & & 2 & 2 \\
\hline UNIFESP & 1 & 3 & 4 \\
\hline UNIFOR & & 2 & 2 \\
\hline UNIGRANRIO & & 1 & 1 \\
\hline UNILASALLE & 1 & & 1 \\
\hline UNIOESTE & & 4 & 4 \\
\hline USP & 10 & 12 & 22 \\
\hline UTFPR & & 3 & 3 \\
\hline Total Geral & 79 & 235 & 314 \\
\hline
\end{tabular}

Fonte: Base das publicações BDTD. 


\section{Considerações Finais}

O mapeamento referente à pesquisa realizada na Biblioteca Brasileira de Teses e Dissertações (BDTD), permitiu investigar a presença da temática de "Educação Inclusiva" e "crianças", nas produções científicas em programas de pósgraduação stricto sensu em nível de mestrado e doutorado nas Universidades Brasileiras, no período de 2000 a 2020.

A análise de conteúdo das teses e dissertações investigadas nesta pesquisa corroborou a percepção de que existe uma vasta produção no cenário nacional de trabalhos que tratem especificamente da Educação Inclusiva. Destaca-se que dos dois tipos de pós-graduação Strito Sensu, o mestrado acadêmico apresentou um número maior de produção cientificas (235) e a menor quantidade de pesquisas sobre o tema foram produzidas pelo Doutorado (79).

Este mapeamento evidenciou a necessidade de aprofundar-se na continuidade desta pesquisa para poder ampliar o estudo referente aos dados de análise sobre esta temática. Porém, fica demonstrado pelos números expressos nos resultados desta pesquisa que há um declínio nas produções referentes à temática proponente nos últimos anos, corroborando para a relevância de se fomentarem futuras pesquisas que incluam essa temática, pois os números ainda são incipientes. Ressaltamos que nessa análise não foram contabilizados os artigos que são derivados das dissertações e teses que colaboram para ampliação desta temática.

A literatura e os resultados deste estudo apontam ainda que devido ao recente surgimento do COVID-19 existe a necessidade de pesquisar a temática relacionada ao desenvolvimento da aprendizagem de alunos especiais durante as aulas ocorridas na referida pandemia (Borges, Bandeira \& Corrêa, 2020) bem como o aprofundamento de estudos que averíguem a inclusão de pessoas com deficiência no ensino superior (Gomes et al., 2021).

\section{Referências}

Albuquerque, A. P. (2005). A subjetividade social de uma escola inclusiva: um estudo de caso. (Dissertação de Mestrado). Faculdade de Educação, Universidade de Brasília.

Almeida, L. M., Furtado, M. A.S \& Yamaguchi, K. K. L. (2020). Educação Inclusiva: um Panorama Sobre o Desenvolvimento e Efetivação do Ensino Inclusivo no Interior do Amazonas. Revista Prática Docente. 5 (2), 1428-1448.

Barbosa, D. S., Fialho, L. M. F. \& Machado, C. J. S. (2018). Educação inclusiva: aspectos históricos, políticos e ideológicos da sua constituição no cenário internacional. Revista Electrónica “Actualidades Investigativas en Educación”, 18 (2).

Bardin, L. (2016). Análise de conteúdo. Edições 70.

Brasil. (2018). Ministério da Educação. Política nacional de educação especial na perspectiva da educação inclusiva. MEC/SEESP.

Borges, L., Bandeira, D. P., \& Corrêa, S. B. C. C. (2020). Inclusão Digital E O Precário Ensino Remoto Em Tempos De Pandemia. III Seminário Diálogos sobre EAD.

Cavalleiro, E. (2006). Introdução. In: Brasil. Ministério da Educação/ Secretaria da Educação Continuada, Alfabetização e Diversidade. Orientações e ações para a educação das relações étnico-raciais. Secad.

Dupin, A. A. da S. Q. \& Silva, M. O. da. (2020). Educação especial e a legislação brasileira: Revisão de literatura. Scientia Vitae, 10 (29).

Ferreira, M. E. C. \& Guimarães, M. (2006). Educação Inclusiva. (2a ed.), DP\&A.

González, J. A. T. (2002). Educação e diversidade: bases didáticas e organizativas, Editora Artmed.

Gomes, E. P., Mendes, J. M. R., Almeida, J. R. S., Duarte, H. A., Vieira, V. R. \& Freire, M. A. M. (2021). Processos de inclusão de pessoas com deficiência no Ensino Superior: Uma revisão sistemática. Research, Society and Development, 10 (8), e11910816977.

Laplane, A. (2006). Uma análise das condições para a implementação de políticas de educação inclusiva no Brasil e na Inglaterra. Educação e Sociedade, CEDES, 27 (96). Edição especial.

Lei no 8.069, de 13 de julho de 1990. Dispõe sobre o Estatuto da Criança e do Adolescente e dá outras providências. Diário Oficial [da] República Federativa do Brasil, Brasília, DF.

Lourenço, E. (2010). Conceitos e práticas para refletir sobre a educação inclusiva. Autêntica.

Matczak, S. A. (1971). Research and composition in philosophy. (2a ed.), Béatrice/Neuwelaerts, 88 p. 
Research, Society and Development, v. 10, n. 10, e282101018961, 2021

(CC BY 4.0) | ISSN 2525-3409 | DOI: http://dx.doi.org/10.33448/rsd-v10i10.18961

Mantoan, M. T. E. (2006). Igualdade e diferenças na escola como andar no fio da navalha. Educação (PUC/RS), XXIX, (58), 55-64.

Mantoan, M. T. E. (2006). Inclusão escolar: pontos e contrapontos, Rosangela Gavioli Prieto: Valeria Amorim Arantes (Org.). (5a ed.), Summus.

Miranda, F. D. (2019). Aspectos Históricos da Educação Inclusiva no Brasil. Revista Pesquisa e Prática em Educação Inclusiva, 2 (3).

Neto, A.O.S., Ávila, E. G., Sales, T. R. S., Amorim, S. S., Nunes, A. K. \& Santos, V. M. (2018). Educação Inclusiva: uma escola para todos. Revista Educação Especial, v. 31(60), Santa Maria. p. 81-92.

Sassaki, R. K. (1997). Construindo uma sociedade para todos. Rio de Janeiro; WVA, Revista Nova Escola.

Severino, A. J. (2017). Metodologia do trabalho científico, livro eletrônico, (2a ed.), Cortez.

Silva, G. F. da, Pletsch, M. D., Sardagna, H. V. \& Bezerra, A. C. S. (2020). Educação Especial e diversidades: emergências atuais. Revista RECC, Canoas, v. $25(1), 7-14$. 